\title{
Rituximab in a Refractory Case of Thrombotic Thrombocytopenic Purpura Associated with Systemic Lupus Erythematosus- Case Report
}

\author{
Khawla K Alghanim $^{1, *}$, Batol G Gasmelseed ${ }^{2}$, Farhan Hameed ${ }^{3}$ and Rehab Y Al-Ansari ${ }^{4}$ \\ ${ }^{1}$ Rheumatology Department, KFMMC, Dhahran 31932, Kingdom of Saudi Arabia \\ IInternal Medicine Department, KFMMC, Dhahran 31932, Kingdom of Saudi Arabia \\ ${ }^{3}$ Nephrology Department, KFMMC, Dhahran 31932, Kingdom of Saudi Arabia \\ ${ }^{4}$ Hematology Department, KFMMC, Dhahran 31932, Kingdom of Saudi Arabia
}

${ }^{*}$ Corresponding author: Khawla K Alghanim, Rheumatology Department, KFMMC, Dhahran 31932, Kingdom of Saudi Arabia, E-mail: dr.khawla25@ yahoo.com

Received: 22 May, 2019 | Accepted: 03 Jun, 2019 | Published: 10 Jun, 2019

Citation: Alghanim KK, Gasmelseed BG, Hameed F, Al-Ansari RY (2019) Rituximab in a Refractory Case of Thrombotic Thrombocytopenic Purpura Associated with Systemic Lupus Erythematosus- Case Report. Autoimmun Infec Dis 3(1): dx.doi.org/10.16966/2470-1025.124

Copyright: (c) 2019 Alghanim KK, et al. This is an open-access article distributed under the terms of the Creative Commons Attribution License, which permits unrestricted use, distribution, and reproduction in any medium, provided the original author and source are credited.

\begin{abstract}
A 34 year- old - pregnant lady, in her 3rd trimester (32 weeks), presented to our emergency department with severe headache. Her clinical examination findings exhibited pallor, malar rash, petechial rash and high blood pressure. Routine blood work revealed leukopenia, lymphopenia, anaemia, and thrombocytopenia in addition to presence of protein in her urine, which later proven to be more than 5 grams in 24 hours urine collection. While her serology was pending she was treated as preeclampsia and delivered by Cesarean section. Subsequently her serology results showed ANA of1: 1260, dsDNA 1:1260 and low complements level. We started her on pulse steroid followed by high dose steroid accompanied by hydroxychloroquine and mycophenolate mofetil. Renal biopsy was done after optimization of her platelets $>50$ which was reported as class 4 lupus nephritis with features suggestive of renal thromboticmicroangiopathy. Peripheral blood smear revealed numerous schistocytes. The patient fulfilled the criteria of systemic lupus erythematosus (SLE) and thrombotic thrombocytopenic purpura (TTP). Unfortunately, she didn't respond to the previous therapies instead her kidney function deteriorated and blood components were coming down further, at that time we started her on daily plasma exchange for total of 7 sessions and she got 5 dose of IV Ig at dose of $400 \mathrm{mg} / \mathrm{kg}$ daily with no significant improvement. Ultimately, We decided to give her 4 doses of Rituximab ( $375 \mathrm{mg} / \mathrm{m} 2$ IV weekly)along with plasma exchange. Fortunately, two days after the first dose of Rituximab, she started to show some improvements in terms of kidney function and rise in her blood components until she was discharged from the hospital with almost normal blood result. Thrombotic thrombocytopenic purpura can happen in the systemic lupus erythematosus and it can be the initial presentation of systemic lupus erythematosus. Rituximab can be used in resistant cases that did not respond to the usual treatments.
\end{abstract}

Keywords: Systemic lupus erythematosus; Thrombotic thrombocytopenic purpura

\section{Introduction}

Thrombotic thrombocytopenic purpura (TTP) is a serious and life threatening condition with a significant morbidity and mortality. Most of the cases of TTP are idiopathic, but some cases may be secondary to connective tissue diseases especially with SLE. TTP in patients with SLE is extremely rare. The overall incidence of TTP in SLE patients has been reported to be as low as $0.5 \%$ [1]. TTP associated with SLE may be refractory to the usual treatments with plasma exchange, and requiring immunosuppressive therapy like high dose corticosteroids and may be cyclophosphamide or mycophenolate mofetil. Plasma exchange should continue to be the first-line treatment for patients with TTP and even to be continued despite adding second line therapy. Appropriate salvage treatment should be started without delay in refractory cases. High dose immunoglobulin infusions may represent a useful combination treatment option with plasma exchange. In addition, these treatments may facilitate recovery and reduce the plasma exchange requirements. We describe a female patient with TTP and SLE who was refractory to plasma exchange, high dose corticosteroids, IV Immunoglobulin and mycophenolate mofetil but responded very well to anti-CD20 antibody (Rituximab) with continues remission after 6 months of follow-up at rheumatology clinic. We reviewed the literature of the previous clinical cases with similar presentation of TTP in SLE. We also provided the appropriate management in such refractory cases, as there is no firm guideline in the literature.

\section{Case Report}

\section{Patient information}

Our patient is a 34 years old pregnant lady in her third trimester (32-weeks' gestation), who has a free medical background. She used 
to follow up with her antenatal care clinic with no complications. She presented to the emergency room (ER) with headache and dizziness for one-day duration associated with some nausea. In the ER department, the obstetric team observed her and decided to admit her for possible C-section.

\section{Clinical examination}

Her blood pressure was high with reading of $175 / 90$ and she was found to be pale with apparent malar rash as well as multiple petechial rashes all over the upper extremities and trunk. She also has a thin hair and significant lower limbs edema.

\section{Diagnostic assessment}

Laboratory test showed anemia, thrombocytopenia and lymphopenia (Table 1) along with significant proteinuria in urine analysis and confirmed by 24 urine collection of protein.

\section{Therapeutic Intervention}

The patient was admitted to the obstetric ward as a case of preeclampsia with possibilities of HELLP syndrome. She was started on anti-hypertensive but unfortunately her blood pressure readings continued to increase and ultimately she was delivered by emergency cesarean section a healthy $2 \mathrm{~kg}$ baby girl. After delivery, she persisted to have high blood pressure and further investigation revealed pancytopenia and significant proteinuria of 5 grams $/ 24$ hours. Hematology and Rheumatology services were involved. Her serology exhibited a positive ANA, positive ds- DNA, low C3 and C4 (Table 1). The diagnosis of Systemic lupus erythematous was made and she was started on pulse steroid and mycophenolate mofetil mainly for her possible proliferative lupus nephritis. The decision of taking kidney biopsy was deferred because of very low platelets which taken later after the platelets transfusion. Daily assessment of the patient showed no improvement instead she developed severe headache again associated with significant blurring of vision while kidney function deteriorated and platelets dropped further to be less than 20 especially after platelet transfusion. At that time, kidney biopsy was reported as class 4 lupus nephritis with thrombotic microangiopathy (Figure 1) and peripheral blood smear showed numerous schistocytes (Figure 2). We diagnosed her to have TTP associated with SLE and immediately we started her on daily plasma exchange and as she continued to have sever thrombocytopenia. We added intravenous immunoglobulin (IVIG) for total of 2 gm over 5 days. We labeled her as refractory TTP

Table 1: Laboratory work upon diagnosis of systemic lupus erythmatosus, after standard therapy and after starting rutixmab

\begin{tabular}{|c|c|c|c|c|}
\hline & Upon Diagnosis & $\begin{array}{l}\text { After Standard } \\
\text { Treatment }\end{array}$ & After Retuximab & Reference range \\
\hline \multicolumn{5}{|l|}{ Hematology } \\
\hline $\mathrm{Hgb}(\mathrm{g} / \mathrm{dl})$ & 7.26 & 6.02 & 7.19 & $13-18$ \\
\hline WBC $\left(10^{\wedge} 3 / \mu \mathrm{l}\right)$ & 7.01 & 3.76 & 4.38 & $4-11$ \\
\hline Lymphocytes $\left(10^{\wedge} 3 / \mu \mathrm{l}\right)$ & 0.833 & 0.50 & 0.90 & $1.5-4$ \\
\hline Platelets & 71 & 18 & 140 & $150-450$ \\
\hline PT INR & 0.86 & 0.85 & 0.92 & $0.8-1.1$ \\
\hline PT (seconds) & 9.80 & 10.9 & 10.7 & $10.9-13.6$ \\
\hline \multicolumn{5}{|l|}{ Chemistry } \\
\hline Creatinine ( $\mu \mathrm{mol} / \mathrm{L})$ & 82 & 205 & 99 & $71-115$ \\
\hline BUN (mmol/L) & 7.6 & 12.4 & 9.6 & $2.5-6.4$ \\
\hline $\mathrm{Na}(\mathrm{mmol} / \mathrm{L})$ & 137 & 141 & 141 & $136-145$ \\
\hline $\mathrm{K}(\mathrm{mmol} / \mathrm{L})$ & 3.0 & 2.4 & 3.4 & $3.5-5.1$ \\
\hline Albumin (g/L) & 12 & 28 & 35 & $34-50$ \\
\hline \multicolumn{5}{|l|}{ Serology } \\
\hline ANA & $1: 1260$ & - & - & Negative \\
\hline Anti-ds-DNA & $1: 1260$ & - & - & Negative \\
\hline Lupus anticoagulant Abs & Negative & - & - & Negative \\
\hline Anti-cardiolipinAb & Negative & - & - & Negative \\
\hline Anti-b2-glycoprotein Ab & Negative & - & - & Negative \\
\hline $\mathrm{C} 3(\mathrm{~g} / \mathrm{L})$ & 0.19 & - & - & $0.9-1.8$ \\
\hline $\mathrm{C} 4(\mathrm{~g} / \mathrm{L})$ & 0.067 & - & - & $0.1-0.4$ \\
\hline CRP & Negative & - & 0.13 & $<6$ \\
\hline 24-h urine protein $(\mathrm{g} / 24 \mathrm{~h})$ & 5 & - & - & - \\
\hline
\end{tabular}

Hgb-hemoglobin, WBC- white blood cells, PT/INR- prothrombin time and international normalized ratio, BUN-blood urea nitogen, Na-sodium, K-POTASSIUM, ANA- antinuclear antibody, ds-DNA- double stranded DNA, C3 -complement 3, C4- complement 4, CRP- C-reactive protein. 


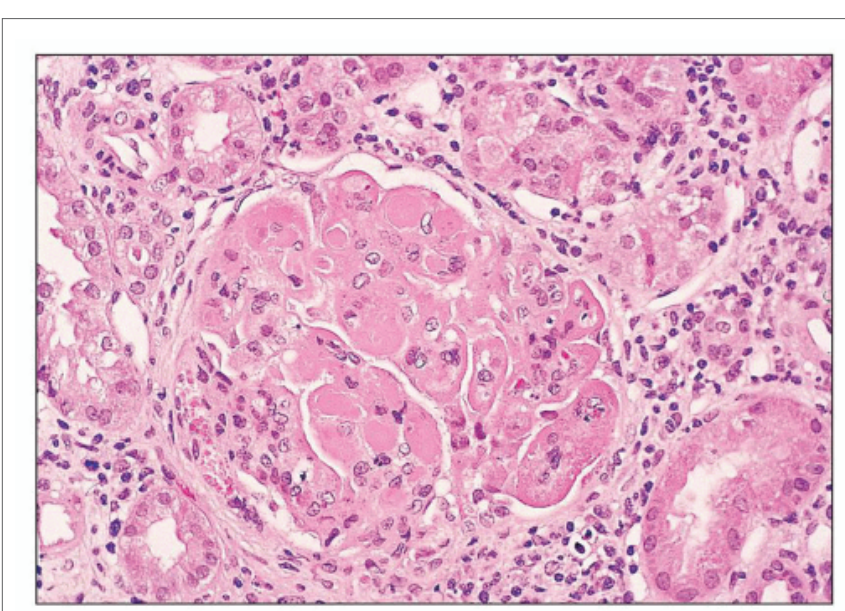

Figure 1: Kidney biopsy showing class 4 lupus nephritis with thrombotic Microangiopathy

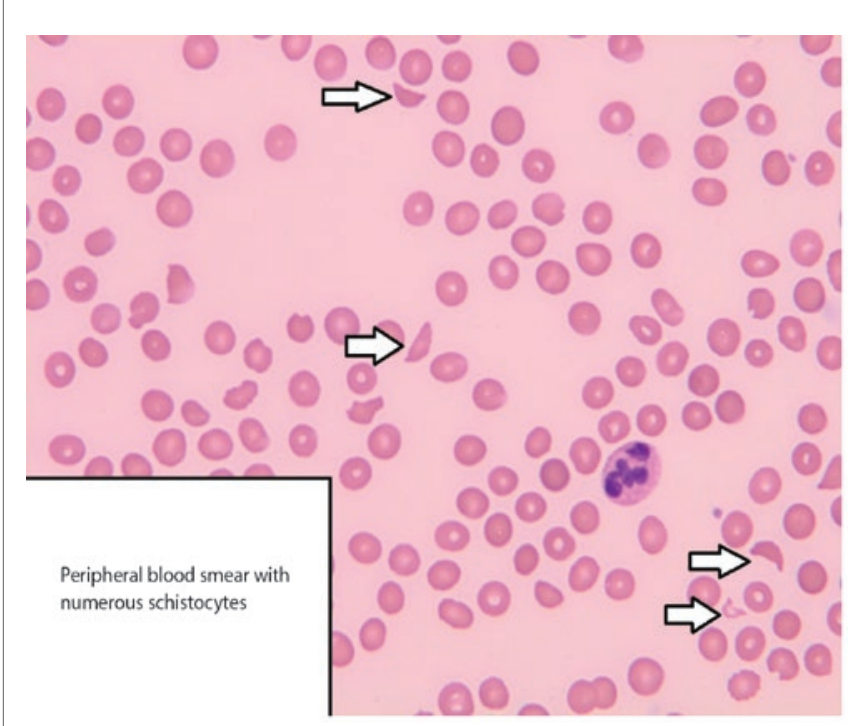

Figure 2: Peripheral blood smear with numerous schistocytes

as she failed to respond to 7 sessions of plasmapheresis. Her disease course was complicated with central line infection which makes her TTP getting worse despite starting her on appropriate antibiotics. We elected to start her on rituximab as 4 doses of rituximab $(375 \mathrm{mg} /$ $\mathrm{m}^{2}$ weekly). Fortunately after first dose of rituximab, her symptoms relieved completely and her platelets and kidney function continued to improve every day until they completely normalized. She was discharged home in a good condition.

\section{Follow up and outcomes}

She continues to follow up at rheumatology clinic with normal platelets counts and kidney function test 6 months after rituximab with no relapses.

\section{Discussion}

Thrombotic thrombocytopenic purpura (TTP) is extremely uncommon in context of connective tissue diseases and the overall incidence has been reported to be as low as $0.5 \%$. The main feature of TTP is microangiopathic haemolytic anemia which is manifested as normocytic anemia, thrombocytopenia, elevated $\mathrm{LDH}$, reduced haptoglobin, elevated unconjugated bilirubin in addition to schistocytes, or fragmented red cells in the peripheral blood smear [1]. SLE can present with hemolytic anemia, thrombocytopenia, neurologic deficits, fever, and renal insufficiency, which is the same presentation of TTP. Subsequently, it makes the diagnosis of TTP in SLE is very challenging topic. However, presence of fragmented RBC's or schistocytes favors the diagnosis of TTP [2]. Active SLE or having renal involvement have increased the risk for developing TTP and the combination of SLE and TTP can be fatal that necessitate rapid and proper management especially in the presence of severe infection [3]. Some data suggest that patients with higher titers of anti-ADAMTS13 autoantibodies are at greater risk for complications and death. Plasma exchange is essential treatment and the replacement with one of the plasma volume like freshfrozen plasma, 24-hour plasma, cryoprecipitate-poor plasma appear to have equivalent efficacy.

The British Committee for Standards in Hematology (BCHS) guidelines as well as American Society of Apheresis Consensus Conference on TTP recommend that plasma-exchange therapy can be continued for a minimum of two days after the platelet count returns to normal $(>150,000 / \mathrm{cu} . \mathrm{mm})$, normal neurological status, rising hemoglobin and normal LDH. The presence of minimum schistocytes on peripheral blood smear after normalization of platelet counts is common and is not predictive of a relapse [4]. After starting plasma exchange, the next step is whether to begin systemic corticosteroids. Corticosteroids are used in the acute management of acquired TTP, and should be started upfront together with Plasma exchange. The stander-starting dose is prednisone $1 \mathrm{mg} / \mathrm{kg}$ per day with exceptional to refractory or critical ill patient with the need of higher doses like pulse therapy of methylprednisolone as $1 \mathrm{~g}$ intravenously once daily for 3 to 5 days. The response to plasma exchange, with or without corticosteroids, is judged by the platelet count. An increased platelet count is anticipated after the second or third daily treatment, and often the platelet count reaches normal in 1 week. For refractory and critically ill patients, twice-daily plasma exchange, each procedure replacing one plasma volume, appears to provide additional benefit when recurrent thrombocytopenia occurs while the patient on plasma exchange, the most important step is to exclude sepsis related to the plasma exchange catheter [5]. Refractory TTP is defined as a failure of platelet response after 4 to 7 days of Plasma exchange, or a clinical deterioration in a patient receiving standard therapy like in our patient. If all of these treatments seem insufficient, additional immunosuppression with cyclophosphamide, vincristine, or cyclosporine maybe started. Although splenectomy is still an option as treatment for patients with refractory or relapsing TTP in some center, we have not done a splenectomy for our patient. There are many reports supporting High-dose immunoglobulin infusion as an effective salvage treatment for cases of TTP refractory to plasma exchange. Furthermore, the literature showed that the use of IVIg is associated with significant reduction in SLE disease activity and improvement in complement levels [6]. For that evidence, we considered adding a high dose IV Ig infusion $(2 \mathrm{gm} / \mathrm{kg})$ in our case. However, our patient did not respond to IV Ig infusion.

Rituximab is a chimeric anti-CD20 monoclonal antibody that depletes B cells, including memory B cells. The study showed the efficacy of rituximab in refractory, relapsing, or new-onset lupus manifestations. As well, there was several studies measured the superiority of using rituximab in duration or response in lupus nephritis in comparison to other immune suppression alone. 
Rituximab may also be given in TTP associated with SLE or refractory primary TTP to plasma exchange, using the conventional regimen of $375 \mathrm{mg} / \mathrm{m}^{2}$ weekly for 4 weeks. Relapse rarely occurs in patients without severe ADAMTS13 deficiency [7].

\section{Conclusion}

In cases of refractory thrombotic thrombocytopenic purpura associated with SLE, rituximab is a good and safe option.

\section{Acknowledgement}

There is no acknowledgement

\section{Conflict of Interest}

The authors declare that there are no conflicts of interest regarding the publication of this paper.

\section{References}

1. Daniel Blum, Geoffrey Blake (2015) "Lupus-associated thrombotic thrombocytopenic purpura-like microangiopathy." World J Nephrol 4: 528-531.
2. Pratish George Jasmine Das, Basant Pawar, Naveen Kakkar (2008) "Thrombotic thrombocytopenic purpura and systemic lupus erythematosus: Successful management of a rare presentation." Indian J Crit Care Med. 12: 128-131.

3. Kwok SK, Ju JH, Cho CS, Kim HY, Park SH (2009) Thrombotic thrombocytopenic purpura in systemic lupus erythematosus: risk factors and clinical outcome: a single center study. Lupus 18: 16-21.

4. Kremer Hovinga JA, Vesely SK, Terrell DR, Lämmle B, George Jv. (2010) Survival and relapse in patients with thrombotic thrombocytopenic purpura. Blood 115: 1500-1511.

5. Vesely SK, George JN (2003) Response: Thrombotic thrombocytopenic purpura-hemolytic uremic syndrome (TTP-HUS): treatment, mortality, and the frequency of HIV infection. Blood 102: 3848-3849.

6. Pauline Anderson, Contributing Writer, MedPage Today (2015) "A review of studies on the effectiveness of rituximab in treating SLE."

7. Karimifar M (2012) Thrombotic thrombocytopenic purpura treated with rituximab in systemic lupus erythematosus. J Renal Inj Prev 1: 53-54. 